\title{
FINITE DIMENSIONAL RINGS OF QUOTIENTS
}

\author{
H. L. HUTSON
}

Dedicated to G.C.

Abstract

In this paper we characterize commutative rings with finite dimensional classical ring of quotients. To illustrate the diversity of behavior of these rings we examine the case of local rings and FPF rings. Our results extend earlier work on rings with zerodimensional rings of quotients.

In this paper we show how the structure of $\operatorname{Spec}_{n}(A)=\{P \mid P \in$ $\operatorname{Spec}(A)$, height $(P) \leq n$ and $P \subseteq$ the zero divisors of $A\}$ determines the Krull dimension of the classical ring of quotients of a commutative ring $A\left(Q_{\mathrm{cl}}(A)\right)$. Our results extend those of $\{\mathbf{H}]$.

In the following all rings are commutative with unit. We let $D(F)=$ $\{P \in \operatorname{Spec}(A) \mid P \not \supset F\}$ whenever $F$ is a subset of $A$. We also set $D^{n}(F)=$ $D(F) \cap \operatorname{Spec}_{n}(A)$. The proof of our first result is a reformulation of $1 \Leftrightarrow 2$ of Theorem 2.1 of $[\mathbf{H}]$.

\section{Theorem 1.}

If $A$ is a commutative ring the following are equivalent

1) $\operatorname{Dim}\left(Q_{\mathrm{cl}}(A)\right) \leq n$.

2) a) $\operatorname{Spec}_{n}(A)$ is compact in the Zariski topology

b) if a finitely generated ideal $I \subseteq \bigcup_{P \in \operatorname{Spec}_{{ }_{n}}(A)} P$ then in fact $I \subseteq$ $P$ for some $P \in \operatorname{Spec}_{n}(A)$.

c) $Z d(A)=$ zero divisors of $A=\bigcup_{P \in \operatorname{Spec}_{n}(A)} P$. 


\section{Proof:}

$1 \Rightarrow 2$. This follows easily from the fact that in this case $\operatorname{Spec}_{n}(A)$ can be identified with $\operatorname{Spec}\left(Q_{\mathrm{cl}}(A)\right)$.

$2 \Rightarrow 1$. Let $Q=Q_{\mathrm{cl}}(A)$. If $\operatorname{dim}(Q)>n$ choose a prime $\mathcal{P} \in \operatorname{Spec}_{n}(Q)$ with height $(\mathcal{P})>n$ then $\mathcal{P} \cap A \supset P_{0}$ with height $\left(P_{0}\right)=n$. Choose $x_{0} \in$ $\mathcal{P} \cap A-P_{0}$. We have $\operatorname{Spec}_{n}(A)=D^{n}\left(x_{0}\right) \cup\left(\bigcup_{x \in P_{0}} D^{n}(x)\right)$. Choose a finite sub cover. $\operatorname{Say} \operatorname{Spec}_{n}(A)=D^{n}\left(x_{0}\right) \cup\left(\bigcup_{i=1}^{n} D^{n}\left(x_{i}\right)\right)$, then $J=\sum_{i=0}^{n} A x_{i}$ is not contained in any prime consisting of zero divisors with height $\leq n$. Hence $J \not \subset \quad \bigcup \quad P$ and thus $J$ contains a regular element. And so $P \in \operatorname{Spec}_{n}(A)$ $J Q=Q$ but $J Q \subset \mathcal{P}$ which is a contradiction.

We now give an application to local rings. A ring is local if it has a unique maximal ideal. We do not require any Noetherian hypothesis. The following result uses a prime avoidance result due to Sharp and Vamos [S\&V].

\section{Corollary 1.}

If $(A, M)$ is a local ring with uncountable residue field and if $\operatorname{Spec}_{n}(A)$ is countable then the following are equivalent

1) $\operatorname{Dim}\left(Q_{\mathrm{cl}}(A)\right) \leq n$.

2) a) $\operatorname{Spec}_{n}(A)$ is compact.

$$
\text { b) } Z d(A)=\underset{P \in \operatorname{Spec}_{n}(A)}{\bigcup} P \text {. }
$$

Proof:

We show that if $I$ is a finitely generated ideal then $I \subseteq \bigcup_{P \in \operatorname{Spec}_{n}(A)} P \Rightarrow$ $I \subseteq P$ for some $P \in \operatorname{Spec}_{n}(A)$. This follows from Proposition 2.5 of [S\&V]: Let $x_{1}, \ldots, x_{k}$ generate $I$ and choose an uncountable family $\left\{u_{\lambda}\right\}, \lambda \in \Lambda$ such that $u_{\lambda}-u_{\nu}$ is a unit when $\lambda \neq \nu$. This is possible because $A / M$ is uncountable. For each $\lambda \in \Lambda$ let

$$
y_{\lambda}=x_{1}+u_{\lambda} x_{2}+\cdots+\left(u_{\lambda}\right)^{k-1} x_{k} \in I \subseteq \bigcup_{P \in \operatorname{Spec}_{n}(A)} P
$$

Since $\operatorname{Spec}_{n}(A)$ is countable and $\Lambda$ is uncountable there is an infinite subset of $\Lambda$ for which $y_{\lambda} \in P_{0}$ for some $P_{0}$ in $\operatorname{Spec}_{n}(A)$. Thus there is a set $\lambda_{1}, \ldots, \lambda_{k}$ with $y_{\lambda_{1}}, \ldots, y_{\lambda_{k}} \in P_{0}$. The $k \times k$ matrix $B=\left(\left(u_{\lambda_{i}}\right)^{j-1}\right)$ 
has determinant which is a unit of $A$ (it is a Vandermonde determinant) and thus $B$ is invertible. We have

$$
B\left(x_{1}, \ldots, x_{k}\right)^{T}=\left(y_{\lambda_{1}}, \ldots, y_{\lambda_{k}}\right)^{T} .
$$

Now applying $B^{-1}$ to both sides of $(*)$ we find that $x_{i} \in P_{0}$ for $i=$ $1, \ldots, k$ and so $I \subseteq P_{0}$.

Note that this result continues to hold whenever $\operatorname{Card}(A / M)>$ $\operatorname{Card}\left(\operatorname{Spec}_{n}(A)\right)$.

In another direction we now consider FPF (finitely pseudo Frobenius) rings. A ring $A$ is FPF if each finitely generated faithful $A$-module generates the category of modules over $A$. Faith $[\mathbf{F}]$ has shown that the following are equivalent:

1) $A$ is FPF.

2) a) $Q_{\mathrm{cl}}(A)$ is self injective.

b) each finitely generated faithful ideal of $A$ is projective.

In the following for a ring $A$ we let $\mathcal{O}_{A}$ denote the structure sheaf of $A$. Also if $S \subseteq \operatorname{Spec}(A)$ then $\Gamma(S, \mathcal{O})$ denotes the ring of sections of $\mathcal{O}$ defined over $S$. Our purpose in the next result is to show that FPF rings with finite dimensional classical ring of quotients enjoy an important property first observed by Deligne: Rings of quotients are often equal to rings of sections. We first became aware of this idea through Lazard $[\mathrm{L}]$. Proofs and additional results along these lines can be found in $[\mathrm{C}]$ and [V].

\section{Theorem 2.}

If $A$ is FPF then the following are equivalent

1) $\operatorname{Dim}\left(Q_{\mathrm{cl}}(A)\right) \leq n$.

2) a) $\operatorname{Spec}_{n}(A)$ is compact.

b) $A=\Gamma(\operatorname{Spec}(A), \mathcal{O}) \rightarrow \Gamma\left(\operatorname{Spec}_{n}(A), \mathcal{O}\right)$ (restriction) is 1-1.

In this case $Q_{\mathrm{cl}}(A) \cong \Gamma\left(\operatorname{Spec}_{n}(A), \mathcal{O}\right) \cong \lim _{\rightarrow}(\operatorname{Hom}(J, A))$ where $J$ varies in the downward directed set of finitely generated and foithful ideals of $A$.

Proof:

$1 \Rightarrow 2$. Compactness follows as before. For (b) let $a \in A$ be identified with a global section of $\mathcal{O}$. If $a \mid \operatorname{Spec}_{n}(A)=0$ then let $Q=Q_{\mathrm{cl}}(A)$ and 
choose any $\mathcal{P} \in \operatorname{Spec}(Q)=\operatorname{Spec}_{n}(Q)$. We have $\mathcal{P} \cap A=P \in \operatorname{Spec}_{n}(A)$ hence there exist $\mu \in A-P$ with $\mu a=0$. It follows that the image of $a$ in $Q_{\mathcal{P}}$ for any prime of $Q$ is zero thus $a=0$ as required.

$2 \Rightarrow 1$. Note that (a) and (b) of two are inherited by $Q$. Assume that there is a prime $P \in \operatorname{Spec}(Q)$ with height $(P)>n$ then the open set $D(P) \supseteq \operatorname{Spec}_{n}(Q)$. By compactness there exists a finitely generated ideal $I \subseteq P$ with the same property. It follows from (b) that $I^{\perp}=\{x \in$ $Q \mid x I=0\}=0$. Since $Q$ is self injective we have by the double annihilator condition. (This is part of a theorem due to Ikeda and Nakayama). See [S, page 274, Prop. 2.1] that $Y^{\perp \perp}=Q$. This is a contradiction.

For the final statement we note that if $I$ is a finitely generated ideal of $A$ then $I$ is faithful $\Leftrightarrow I$ contains a regular element $\Leftrightarrow D(I) \supseteq \operatorname{Spec}_{n}(A)$. To see this observe that if $I$ is f.g. and faithful then $I \operatorname{Hom}(I, A)=$ Hom $(I, A)$ since $I$ is projective (the dual basis lemma). We thus have $I \lim (\operatorname{Hom}(J, A))=\lim _{\rightarrow}(\operatorname{Hom}(J, A))$ and so $\lim (\operatorname{Hom}(J, A))=Q_{\text {tot }}(A)$ (the maximal flat epimorphic extension of $\vec{A}[\mathbf{S}]$ ). Because of this, if a f.g. faithful ideal consists entirely of zero divisors then $Q_{\text {tot }}(A) \neq$ $Q_{\mathrm{cl}}(A)$ but this is not the case since $Q_{\mathrm{cl}}(A)$ is self injective and hence flat epimorphicly closed. The second equivalence is now a consequence of (b). The rsult follows from this obesrvation because $Q_{\mathrm{cl}}(A)$ is the localization of $A$ at the Gabriel filter of ideals containing regular elements [S] and by Theorem 5.24 of $[\mathrm{V}]$ since $\operatorname{Spec}_{n}(A)$ is compact and generically closed.

We add that the proof of this result shows that the condition $(A \rightarrow$ $\Gamma\left(\operatorname{Spec}_{n}(A), \mathcal{O}\right)$ is 1-1) implies $\left.\left.2 b\right)+2 c\right)$ of Theorem 1 for any FPF ring. Thus another proof is available. We choose our statement of Theorem 2 and our approach because of its geometric character.

\section{References}

[C] F. W. CALL, Torsion theoretic algebraic geometry, Queen's papers in Pure and Applied Mathematics 82 (1989).

[F] C. FAITH, "Injective modules and injective quotient rings," Lecture Notes in Pure and Appl, Math. 72, Marcel Dekker, Basel and New York, 1982.

[F1] C. FAITH, "Algebra II; Ring theory," Springer Verlag, Berlin, Heidelberg, New York, 1976.

$[\mathrm{H}] \mathrm{H}$. L. Hutson, On zero-dimensional rings of quotients and the geometry of minimal primes, J. Algebra 112 (1988), 1-14.

[L] D. LAzArd, Autour de la platitude, Bull. Soc. Math. France $\mathbf{9 7}$ (1969), 81-125. 
$[S \& V]$ R. Sharp and P. Vamos, Baire's category theorem and prime avoidance in complete local rings, Arch. Math. 44 (1985), 243-248.

[S] S. STENStrom, "Rings of quotients," Springer Verlag, Berlin, Heidelberg, New York, 1975.

[V] A. Verschoren, "Relative invariants of sheaves," Marcel Dekker Inc., New York and Bassel, 1987.

Department of Mathematics

The U.S Merchant Marine Academy

Kings Point N.Y. 11024

U.S.A.

Primera versió rebuda el 17 de Novembre de 1992 , darrera versió rebuda el 20 de Gener de 1993 